\title{
UN PROYECTO PARA TRABAJAR LA INTELIGENCIA ESPIRITUAL A TRAVÉS DE COACHING
}

\section{AN APPROACH TO WORKING SPIRITUAL INTELLIGENCE THROUGH COACHING TECHNIQUES}

Elena Alarcón Orozco¹. Universidad de Málaga (CAMMIA). España. elenaalarcon@eummia.es

\section{RESUMEN}

En pleno siglo XXI y ante el momento social que estamos viviendo, nos planteamos como reto educativo trabajar para alcanzar el desarrollo íntegro de la persona, algo que es difícil de conseguir si solo trabajamos las capacidades cognitivas o culturales. A partir de esta idea y tomando como referencia la concepción humanista del ser humano como ser bio-psico-socio-espiritual, nos planteamos como objetivo la formación de docentes preparados para trabajar todas las facetas humanas, esto incluye las capacidades emocionales y espirituales. Utilizando como técnica de investigación el análisis documental, hemos recogido información que nos ha permitido conceptualizar la inteligencia emocional y la inteligencia espiritual desde la perspectiva de la psicología humanista y la psicología positiva, relacionando los componentes de una y otra, de manera que pudiéramos diseñar una propuesta de intervención que nos permita trabajar al mismo tiempo, en el desarrollo de habilidades tanto emocionales como existenciales. En base a este trabajo, hemos diseñado un proyecto de intervención educativa, que utiliza el coaching como instrumento para desarrollar ambas capacidades en el ámbito educativo. De este modo, creemos que, fomentando el desarrollo de las facetas espirituales y las fortalezas humanas, podemos contribuir al desarrollo de competencias y alcanzar así una de las finalidades del sistema educativo.

PALABRAS CLAVE: Inteligencia espiritual, Inteligencia emocional, Coaching, Psicología positiva, Psicología humanista.

\section{ABSTRACT}

Amid the $21^{\text {st }}$ century and in the social moment we are living now, working the complete individual development appears as an educative goal which is difficult to achieve if we only train cognitive and cultural skills. On that basis and from the humanist conception of the individual as a bio-psycho-social-spiritual being, it is our main objective training future teachers ready to deal with all human dimensions, emotional and spiritual capacities included. We have gathered information -using documentary analysis- which have allowed us to contextualize emotional and spiritual intelligences from the point of view of humanist and positive psychology,

\footnotetext{
${ }^{1}$ Elena Alarcón Orozco: Doctora Cum Laude por la Universidad de Málaga. Profesora titular del departamento de Psicología del Centro Adscrito de Magisterio M. ${ }^{a}$ Inmaculada de Antequera. Sus líneas de investigación son las metodologías activas, la resolución de conflictos en el aula derivados del trabajo en equipo y las altas capacidades.
} 
relating components of both, in order to design an action approach that lets us work at the same time- emotional and existential skills. For this project we have planned an educational action that uses coaching techniques as tools for developing both skills in educational environment. Thus, by encouraging the progress of these spiritual dimensions and human strengths, we can provide the upbringing of skills and achieve one of the purposes of the educative system.

KEYWORDS: Spiritual intelligence, Emotional intelligence, Coaching, Positive psychology, Humanistic psychology.

\section{Cómo citar el artículo:}

Alarcón Orozco, E. (2020). Un proyecto para trabajar la inteligencia espiritual a través de coaching. Revista de Ciencias de la Comunicación e Información, 25(2), 91-105. doi: http://doi.org/10.35742/rcci.2020.25(2).91-105

\section{INTRODUCCIÓN}

Uno de los retos que nos plantea el actual sistema educativo es el desarrollo integral de la persona, que conlleva la construcción de su personalidad y el desarrollo máximo de sus capacidades intelectuales, sociales, culturales y emocionales. En este sentido, si buscamos la mejora de la calidad de la enseñanza y el aprendizaje, debemos trabajar en todas y cada una de las dimensiones del ser humano.

Este reto educativo ha propiciado que en la actualidad el interés por las capacidades intelectuales, sociales y culturales ha sido superado por la atención al desarrollo de capacidades emocionales que permiten el conocimiento de sí mismo y de los demás. Sobre todo, tras la publicación de la Teoría de las Inteligencias Múltiples de Gardner y la de la Inteligencia Emocional de Goleman que han influido notablemente en el enunciado de las actuales leyes de educación (LOE y LOMCE). De hecho, ya nadie cuestiona la existencia de la inteligencia emocional ni su importancia, pues está demostrada la correlación entre cognición y emoción.

A través del desarrollo de la inteligencia emocional, podemos ser capaces de reconocer, comprender, facilitar y regular nuestras emociones para afrontar las distintas situaciones que se dan en nuestras vidas (Palomera, Fernández-Berrocal y Brackett, 2008), pero el desarrollo de la inteligencia emocional no es suficiente para aprender a ser o para el desarrollo integro de la persona.

Tal y como apunta Torralba (2018), el hombre no es solo un ser biopsicosocial, sino que existen un conjunto de necesidades en la persona que no son de orden corporal, ni psicológico, ni social, son de orden espiritual, que, aunque se ubiquen en el campo psicológico, exigen el trabajo de una capacidad que va más allá, la Inteligencia Espiritual, para poder darles respuesta satisfactoriamente. En este sentido, la psicología positiva y la psicología humanista presentan una concepción integrada del ser humano como una realidad bio-psico-socio-espiritual (Vargas y Moya, 2018). Esta reciente corriente ha provocado que, en diferentes sectores académicos y profesionales aumente el interés y la concienciación por considerar la 
espiritualidad como una parte esencial e integral del desarrollo de la personalidad (Salgado-Lévano, 2015).

Por otro lado, el aumento del interés por la inteligencia emocional ha provocado que aparezcan multitud de propuestas para promoverla, tanto en el ámbito escolar, como en el laboral, entre ellas el coaching, entendido este como una técnica metodológica que favorece el desarrollo del potencial (Sánchez y Boronat, 2014) o de crecimiento personal. En este trabajo vamos a presentar un acercamiento al estudio de la espiritualidad y la inteligencia espiritual, de manera que incida en el desarrollo integral de la persona y una propuesta de coaching como estrategia para trabajar no solo a nivel emocional, sino también espiritual, fomentando así esta faceta humana.

\section{ESTADO DE LA CUESTIÓN: INTELIGENCIA EMOCIONAL, INTELIGENCIA ESPIRITUAL Y EDUCACIÓN}

Podríamos comenzar este apartado buscando respuestas a un interrogante: ¿de dónde surge el interés por el desarrollo de capacidades diferentes a las intelectuales?

Según Bisquerra (2011, p. 11) "la educación emocional es una respuesta a las necesidades sociales que no están suficientemente atendidas en el curriculum académico ordinario". La organización del sistema educativo debe responder a las demandas sociales. En la actualidad, vivimos en una sociedad globalizada y caracterizada por el cambio y la transformación que se produce a una velocidad vertiginosa, por tanto, la posesión del conocimiento no es tan importante como las habilidades para saber gestionar la información y tomar decisiones que permitan al individuo la rápida adaptación al cambio. Es decir, el sistema educativo debe tener como objetivo, el desarrollo máximo de las capacidades que permitan al individuo adaptarse a los cambios y, como apunta Bisquerra (2011) alcanzar el bienestar individual y social.

Podríamos situar el aumento del interés por el estudio de la inteligencia espiritual y emocional a finales del siglo XX, con la aparición de la teoría de las inteligencias múltiples de Gardner y la difusión de los trabajos de Goleman. Sin embargo, hay un gran desconocimiento social tanto de la inteligencia espiritual como de la emocional, situando a ambas como parte de la teoría de las inteligencias múltiples de Gardner, y en principio no fue así.

Tal y como apunta Woolfolk (2011) Gardner entendía la inteligencia como el resultado de varias capacidades independientes, hasta ese momento consideradas como talentos. La teoría de las inteligencias múltiples de Gardner surge como alternativa cuando este pone en duda el cociente intelectual $(\mathrm{Cl})$ como medida del potencial humano. En su obra, Gardner (1995) distingue siete inteligencias: lingüística, musical, lógico-matemática, espacial, cinestésico-corporal, intrapersonal e interpersonal. En 2001, añade la inteligencia naturalista y aunque especula acerca de la posibilidad de la existencia de la inteligencia espiritual, nunca termina de incluirla en su modelo, pero tampoco descarta la posibilidad de hacerlo en un futuro, pues la considera digna de atención (Gardner, 2011; Arias y Lemos, 2015). De hecho, desde el inicio se refiere a ella como inteligencia existencial o trascendental, 
no como inteligencia espiritual, y la considera como una capacidad central, describiéndola como la capacidad de situarse a uno mismo con las facetas más extremas del cosmo (lo infinito) y la capacidad afín de situarse a uno mismo en relación a determinadas características existenciales de la condición humana (el significado de la vida y la muerte, el destino final del mundo y ciertas experiencias de sentir amor o deleite con el arte y la belleza) (Gardner, 2011).

Con respecto a la inteligencia emocional, Gardner nunca se plantea su existencia como tal, porque, tal y como apunta Bisquerra (2003), considera que está formada por la inteligencia interpersonal e intrapersonal. La primera tiene que ver con la inteligencia social y la segunda con la personal.

Por otro lado, el interés por la inteligencia emocional surgió a raíz de la obra de Goleman (1995), en la que la presenta en contraste con la inteligencia racional, considerando que la primera puede ser tan poderosa o más que la segunda, teniendo en cuenta, además, que las competencias emocionales se pueden aprender $\mathrm{y}$, por tanto, todas las personas podemos ser emocionalmente inteligentes (Bisquerra, 2003). Para Zohar y Marshall (2001), la inteligencia emocional nos hace conscientes de los sentimientos propios y de los demás y nos permite responder de manera adecuada al dolor y al placer.

Nosotros pensamos que ambas inteligencias (emocional y espiritual) están íntimamente relacionadas, pues los componentes de una se enraízan en la otra, no puede ser la una sin la otra y las dos son necesarias para el desarrollo pleno e integro de la persona, por tanto, deben ser trabajadas desde el ámbito educativo.

Desde el punto de vista de la psicología, podríamos situar el origen de la inteligencia espiritual en la psicología humanista, entendida como la tercera fuerza en psicología, después del psicoanálisis y el conductismo. Mientras que el estudio de la inteligencia emocional estaría más relacionado con la psicología positivista, cuarta fuerza en psicología.

No debemos olvidar que, tal y como describen Vargas y Moya (2018), la psicología humanista defiende una concepción humana nada reduccionista, entendiéndolo como un ser bio-psico-socio-espiritual que a medida que madura psicológicamente, tiende a la autoconstrucción y la búsqueda del sentido de su vida, construyendo un proyecto vital en torno al cual giran sus propias características personales. De hecho, Pérez (2016), consideraba a Maslow como un precursor de la descripción de lo que hoy consideramos inteligencia espiritual, ya que entiende la "autorrealización" como un estado espiritual que permite al individuo ser creativo, feliz, tolerante y tener como propósito ayudar a los demás a alcanzar este estado, colocándolo en la cúspide de su pirámide de necesidades humanas.

Para Vargas y Moya (2018), la educación, según la perspectiva humanista, debe facilitar las condiciones que permitan el pleno desarrollo de la persona y esto comprende también el enriquecimiento del componente espiritual.

La psicología positiva está enraizada en la humanista, pero se caracteriza por centrarse en temas relacionados con el bienestar y la felicidad, con el objetivo de 
estudiar las experiencias positivas y rasgos individuales positivos que inciden en la mejora de la calidad de vida, el logro de la felicidad y el bienestar de las personas (Bisquerra, 2013; Vargas y Moya, 2018). Según Bisquerra (2011), la psicología positiva, surge con posterioridad a la educación emocional, pero contribuye a reforzar sus objetivos hacia las emociones positivas y el bienestar, siendo este la finalidad de la educación.

Como podemos deducir, aunque la inteligencia espiritual y emocional pudieran tener el mismo punto de partida, no son lo mismo, pero están íntimamente relacionados. Tanto la perspectiva humanista como la positiva, se plantean como objetivo de la educación el desarrollo pleno de la persona y el aumento de las emociones positivas y el bienestar. Para alcanzar este objetivo, hay que fomentar tanto la inteligencia emocional como la inteligencia espiritual. Zohar y Marshall (2001) van más allá, considerando la inteligencia espiritual como primordial, sería la base para el buen funcionamiento del resto de inteligencias.

\subsection{La espiritualidad como fortaleza humana}

Desde la psicología positiva, la espiritualidad es considerada como una fortaleza humana que se relaciona con un desarrollo pleno y maduro. No podemos obviar que la psicología positiva surge en los años posteriores a la Segunda Guerra Mundial, con el objetivo de centrarse en el potencial humano en lugar de las deficiencias psicológicas, enfatizando las experiencias positivas y los rasgos personales positivos. Tanto para Bisquerra (2011) como para Vargas y Moya (2018), para lograr este objetivo habría que trabajar en el desarrollo de las virtudes y fortalezas que nos van a permitir alcanzar estados positivos como alegría, esperanza, espiritualidad, confianza o serenidad personal (Carr, 2007).

Peterson y Park (2009) y Castro (2010) recogen una propuesta de 24 fortalezas, agrupadas en 6 virtudes y que van a permitir el desarrollo pleno del potencial humano. Según Bisquerra (2011), el desarrollo de estas competencias previene la aparición de trastornos psicológicos. Estas son:

1. Sabiduría y conocimiento: creatividad, curiosidad, apertura de mente, amor al aprendizaje y perspectiva.

2. Coraje: valentía, persistencia, vitalidad y autenticidad.

3. Humanidad: amabilidad o bondad, inteligencia social y amor.

4. Justicia: equidad, liderazgo y trabajo en equipo.

5. Templanza o contención: capacidad de perdonar, autorregulación, prudencia y modestia.

6. Trascendencia: apreciación por la belleza y la excelencia, gratitud, esperanza, humor y espiritualidad.

Todas estas fortalezas conducen a la satisfacción vital, el bienestar psicológico y la felicidad y pueden ser fomentadas y enseñadas (Bisquerra, 2011; Vargas y Moya, 2018).

Para Peterson y Seligman (2004, citados en Vargas y Moya, 2018), la espiritualidad es la fortaleza más humana y la definen como "la capacidad de tener creencias 
coherentes en relación con el propósito más alto, al significado del universo y al lugar que ocupamos en él" (p. 293). Es decir, esta fortaleza hace que uno se haga preguntas sobre el sentido de la vida, de modo que la respuesta influirá en nuestras conductas.

Por otro lado, no debemos olvidar, como recuerda Torralba (2018) que tradicionalmente se ha unido la espiritualidad con la vivencia religiosa, sin embargo, las corrientes más actuales hablan de una espiritualidad laica, reconociendo el valor de lo espiritual en la vida humana y desligándolo de las tradiciones religiosas. En este mismo sentido, Álvarez, (2015) considera la espiritualidad como un proceso de la vida y desarrollo del ser humano que se centra en la búsqueda del sentido de la vida, la moralidad y el bienestar y que influye en las decisiones y en las acciones que realizamos a lo largo de la vida. Para esta autora, la palabra espiritualidad se está utilizando como forma alternativa de religión, quizás porque cuando hablamos de religión, la mayoría basa su opinión teniendo en cuenta sólo el ritualismo externo, pero no podemos obviar que la religión es una forma de expresar y vivir la dimensión espiritual de la persona, por tanto, tenemos la necesidad de cuidar esta dimensión interior en respuesta a la crisis de valores que estamos viviendo.

Podríamos decir que la espiritualidad forma parte de la totalidad del ser, de modo que la integridad espiritual se puede considerar como una necesidad básica humana (Sánchez, 2009) configurándose como una dimensión que permite al ser humano relacionarse consigo mismo, con los demás y con Dios (Salgado-Lévano, 2015).

Independientemente del concepto que tengamos de espiritualidad, todos los autores coinciden en el hecho de que se puede y debe trabajar para el pleno desarrollo de la persona, por tanto, el desafío educativo actual está en cómo formar a educadores de manera que asuman que la dimensión espiritual forma parte del desarrollo evolutivo de todo ser humano. El docente no solo debe estar formado y actualizado en los avances de la ciencia y la inteligencia emocional, sino también en el campo de la espiritualidad. La espiritualidad o competencia espiritual es una dimensión de la persona educable, en este sentido, Álvarez (2015) habla de educación para la interioridad.

Este es el verdadero reto educativo del siglo XXI: fomentar la espiritualidad a través de la inteligencia espiritual, de modo que podemos facilitar el conocimiento de sí mismo y el desarrollo integral de la persona.

\subsection{La inteligencia espiritual}

Como ya apuntábamos más arriba, la inteligencia espiritual no forma parte de la teoría de las inteligencias múltiples de Gardner. Realmente, las primeras autoras en hablar de inteligencia espiritual, desde un punto de vista científico, fueron Zohar y Marshall, considerándola como la "capacidad del ser humano para afrontar y trascender el sufrimiento y el dolor, dar valor, significado y sentido a los actos humanos" (Álvarez, 2015, p. 149), permitiendo a las personas afrontar y resolver problemas de significados y valores, poner nuestros actos y nuestras ideas en un contexto más rico y significativo, y determinar que una opción es más valiosa que otra (Zohar y Marshall, 2001). 
Hay que tener en cuenta que "la falta de inteligencia espiritual conduce al fanatismo, gregarismo, banalidad, consumismo innecesario, vacío existencial, aburrimiento personal, autoengaño e intolerancia" (Bustelo, Miró y Barquero, 2018, p. 44). Tal y como apuntan estos autores, no es fácil observar en escuelas y/o universidades el tratamiento de esta inteligencia, quizás por la visión materialista y el sentido o exigencia del beneficio inmediato que dominan el mundo y los valores humanos actuales. Sin embargo, teniendo en cuenta el modo en que entendemos la inteligencia espiritual, dota al individuo de las herramientas contra esta forma de alineamiento ya que, como afirman Zohar y Marshall (2001), la inteligencia espiritual nos permite afrontar y resolver problemas de significados y valores. Bustelo et al. (2018) consideran que el desarrollo de la inteligencia espiritual es el "antídoto contra los mecanismos de propagación social" (p. 45). Por tanto, hay que fomentarla, algo en lo que llevan trabajando numerosos autores como Torralba (2018) y Arias y Lemos (2015), que estudian los múltiples beneficios que tiene cultivar la inteligencia espiritual para el desarrollo integral de la persona.

Para poder trabajar la inteligencia espiritual, debemos conocerla. Nosotros, para este trabajo, vamos a tomar como referencia el modelo de inteligencia espiritual descrito por Arias y Lemos (2015), que tiene tres dimensiones, cada dimensión tiene seis facetas o aspectos que quedan recogidos en la tabla 1:

- Cognitiva: hace referencia al conocimiento espiritual

- Emocional, entendido como la vivencia espiritual

- Conductual o contingencia que implica la coherencia conductual en función del conocimiento espiritual.

Tabla 1. Facetas que componen la inteligencia espiritual según modelo de Arias y Lemos (2015)

\begin{tabular}{ccc}
\hline $\begin{array}{c}\text { Conocimiento espiritual } \\
\text { (cognitiva) }\end{array}$ & $\begin{array}{c}\text { Vivencia espiritual } \\
\text { (emocional) }\end{array}$ & Contingencia (conductual) \\
\hline Razonamiento moral & Entusiasmo & Control de los impulsos \\
Practica de la meditación & Sensibilidad estética & Sobriedad, sencillez \\
Búsqueda de sentido & Admiración por lo misterioso & Manejo del ocio \\
Autoconocimiento & Empatía & Cuidado de la salud \\
Autotrascendencia & Paz interior & Estoicismo o resiliencia física \\
Actitud frente al dolor & Felicidad & Ejercicio de la solidaridad \\
\hline
\end{tabular}

Fuente: elaboración propia

Esta descripción de la inteligencia espiritual va en línea con la propuesta que en su día hicieron Zohar y Marsahll (2001) considerando tres procesos psicológicos, el proceso primario, haría referencia a lo racional, el secundario a lo emocional y el tercer proceso, el espiritual, unifica, integra y puede transformar la materia que surge de los otros dos que compiten entre sí por el control, y la expresión. Pero además no se alejan de la descripción de las fortalezas humanas aportada por Peterson y Park (2009) y Castro (2010) y recogidas por Vargas y Moya (2018), ya que estas se pueden relacionar con las facetas descritas por Arias y Lemos (tabla 2). 
Tabla 2. Aproximación de la equivalencia entre facetas de la inteligencia espiritual y las fortalezas humanas

\begin{tabular}{cccccc}
\hline $\begin{array}{c}\text { Conocimiento } \\
\text { espiritual } \\
\text { (cognitiva) }\end{array}$ & $\begin{array}{c}\text { Fortalezas } \\
\text { humanas }\end{array}$ & $\begin{array}{c}\text { Vivencia } \\
\text { espiritual } \\
\text { (emocional) }\end{array}$ & $\begin{array}{c}\text { Fortalezas } \\
\text { humanas }\end{array}$ & $\begin{array}{c}\text { Contingencia } \\
\text { (conductual) }\end{array}$ & $\begin{array}{c}\text { Fortalezas } \\
\text { humanas }\end{array}$ \\
\hline $\begin{array}{c}\text { Razonamiento } \\
\text { moral }\end{array}$ & Justicia & Entusiasmo & Persistencia & $\begin{array}{c}\text { Control de los } \\
\text { impulsos }\end{array}$ & Autorregulación \\
\hline $\begin{array}{c}\text { Practica de la } \\
\text { meditación }\end{array}$ & $\begin{array}{c}\text { Apertura de } \\
\text { mente }\end{array}$ & $\begin{array}{c}\text { Sensibilidad } \\
\text { estética }\end{array}$ & $\begin{array}{c}\text { Apreciación } \\
\text { de la belleza }\end{array}$ & $\begin{array}{c}\text { Sobriedad, } \\
\text { sencillez }\end{array}$ & Modestia \\
\hline $\begin{array}{c}\text { Búsqueda de } \\
\text { sentido }\end{array}$ & Curiosidad & $\begin{array}{c}\text { Admiración } \\
\text { por lo } \\
\text { misterioso }\end{array}$ & $\begin{array}{c}\text { Religiosidad } \\
\text { Amor }\end{array}$ & $\begin{array}{c}\text { Manejo del } \\
\text { ocio }\end{array}$ & Autorregulación \\
\hline Autoconocimiento & Autenticidad & Empatía & $\begin{array}{c}\text { Inteligencia } \\
\text { social }\end{array}$ & $\begin{array}{c}\text { Cuidado de la } \\
\text { salud }\end{array}$ & Vitalidad \\
\hline Autotrascendencia & Perspectiva & Paz interior & Amor & $\begin{array}{c}\text { Estoicismo o } \\
\text { resiliencia } \\
\text { física }\end{array}$ & Vitalidad \\
\hline $\begin{array}{c}\text { Actitud frente al } \\
\text { dolor }\end{array}$ & Valor & Felicidad & Gratitud & $\begin{array}{c}\text { Ejercicio de la } \\
\text { solidaridad }\end{array}$ & $\begin{array}{c}\text { Bondad } \\
\text { Perspectiva }\end{array}$ \\
\hline
\end{tabular}

Fuente: elaboración propia

Por tanto, no podemos entender la plenitud de la persona sin hacer referencia a este componente espiritual, sea inteligencia espiritual o existencial, que aúna y nutre las demás facetas e inteligencias del ser humano. Teniendo en cuenta que la espiritualidad es una capacidad que se encuentra repartida de manera desigual, se nutre de diferentes circunstancias y hay que trabajarla para que se desarrolle y madure, tendríamos que pensar en las diferentes técnicas o programas para fomentarla. En este sentido Zohar y Marsall (2001) proponen fomentar la inteligencia espiritual aumentando nuestra tendencia a preguntar por qué, a buscar conexiones entre las cosas, a ser más reflexivos, a ser más consciente con nosotros mismos, más honestos e incluso valientes. Algo que en pleno siglo XXI podemos trabajar a través del coaching.

\section{3. ¿POR QUÉ COACHING?}

Al inicio de este trabajo planteábamos la necesidad de fomentar la inteligencia espiritual desde el ámbito educativo para alcanzar el desarrollo íntegro de la persona y dotarla de herramientas y competencias para gestionar la información de la sociedad del conocimiento.

En este sentido, creemos que el coaching puede ser una forma apropiada de trabajar esta dimensión humana ya que, como afirman Sánchez Mirón y Boronat Mundina (2014), los principios del coaching coinciden con los de nuestro sistema educativo: el desarrollo de competencias, tomando como punto de partida el compromiso durante el proceso de enseñanza-aprendizaje.

Para estas autoras, el coaching es una técnica metodológica que favorece el desarrollo del potencial humano. Para Whitmore (2016, p. 21) el coaching consiste en liberar el potencial de las personas, para que puedan desarrollar al máximo sus capacidades, "consiste en ayudarles a aprender en lugar de enseñarles". Para 
Domínguez, Cruz y Ferrando (2018) es una herramienta de desarrollo personal, basada en el acompañamiento. Mientras que para Ramos, Sierra y Roces (2019), definen el coaching como un método y un proceso para aumentar la calidad de vida y el rendimiento de las personas.

En cualquier caso, el objetivo del coaching, definido como técnica, herramienta o método, no es otro que el crecimiento personal, el desarrollo del potencial humano, que, a fin de cuentas, es el objetivo de nuestro actual sistema educativo.

El coaching como técnica nace en el mundo del deporte y se traslada al mundo empresarial y de las organizaciones, aunque sus principios y fundamentos, tal y como apunta Bisquerra (2008) deben buscarse en la psicología humanista, el existencialismo de Sastre y el construccionismo, entre otras corrientes. Este autor relaciona el coaching con la orientación, fundamentalmente psicopedagógica, que se ha ido abriendo camino en el mundo empresarial, teniendo más éxito en este campo que en el educativo, hasta hace muy poco, que el interés por el coaching educativo ha ido en aumento. Es posible que este haya ocurrido porque la importancia por las competencias emocionales y espirituales, hasta hace muy poco, han estado superadas por las competencias cognitivas y es ahora cuando hay cambio de tendencia.

Particularmente consideramos que el coaching puede ser apropiado para trabajar la inteligencia espiritual por su fundamentación filosófica y psicológica.

Siguiendo a Sánchez y Boronat (2014), dentro de los fundamentos filosóficos hay que destacar la mayéutica socrática. Tanto la base del coaching como de la inteligencia espiritual se apoya en el método socrático: por medio de preguntas, la persona va resolviendo problemas, tomando decisiones de forma autónoma, llegando a descubrir su potencial.

El coaching es descrito por la mayoría de los autores como un proceso dialógico donde se establecen metas y un plan de acción para alcanzarlas. Una conversación entre dos o más personas: coach, persona que ayuda a otra en su crecimiento personal, y coachee, persona que recibe la ayuda (Bisquerra, 2008). El coach hace preguntas para que el coachee encuentre las respuestas y él mismo desarrolle una nueva forma de percibir, sentir y resolver situaciones que, en principio puede considerar problemáticas (Barrón, 2010).

Con respecto a la fundamentación psicológica, ya hemos hablado de la importancia de la psicología humanista en el origen de la inteligencia espiritual, de la misma manera podríamos situarla en el origen del coaching. A esto tendríamos que añadir el construccionismo, que como apuntan Sánchez y Boronat (2014), enfatiza el papel de la interacción entre el ambiente y el individuo en la construcción de la persona.

Teniendo en cuenta estas tres líneas de influencia, podríamos concluir que el coaching se vale de la mayéutica socrática para que, desde una perspectiva humanista y en interacción con el ambiente y contexto que rodea al individuo, este pueda buscar respuestas a preguntas que le van a permitir encontrar sentido a su vida, construir un proyecto vital en torno al cual girarán sus virtudes y fortalezas. 


\subsection{Coaching espiritual como herramienta para trabajar la inteligencia espiritual.}

El coaching se puede aplicar en diferentes contextos, así hemos encontrado trabajos dirigidos a directivos, coaching ejecutivo, a organizaciones, coaching organizacional, en el campo deportivo, coaching deportivo, en el campo nutricional, coaching nutricional, etc. Nosotros, queremos realizar una propuesta de coaching para trabajar la inteligencia espiritual, contribuyendo de este modo al desarrollo íntegro de la persona. Pretendemos desarrollar esta propuesta en el ámbito educativo, de modo que nos podemos basar en el coaching educativo, pero, además, creemos que la inteligencia espiritual y la espiritualidad están íntimamente relacionados con la inteligencia emocional, por tanto, también debemos basar nuestra propuesta en el coaching emocional. De modo que proponemos una estrategia de coaching espiritual como proceso dirigido al crecimiento personal a través de la búsqueda de respuestas que permitan al individuo encontrar el sentido de la vida.

Sea cual sea el campo de aplicación del coaching, todos tienen el mismo objetivo y la misma fundamentación e influencia teórica, compartiendo, por tanto, los pilares o principios del coaching que, según Carril (2008) y Sánchez y Boronat (2014) son:

a) Tomar conciencia de uno mismo y aceptar lo que somos.

b) Marcarse metas motivadoras, salir de la zona de confort.

c) Ser constantes y avanzar poco a poco, siguiendo nuestra intuición y siendo dueños de nuestro tiempo.

d) Reflexionar para reconducirnos y avanzar.

También hemos encontrado en la literatura diferentes modelos o escuelas de coaching que establecen distintas formas de trabajarlo. No es nuestro objetivo hacer una larga exposición sobre este tema, así que nos vamos a centrar en el modelo en el que hemos basado nuestra propuesta y que recogen Sánchez y Boronat (2014) basado en el trabajo de Bayón, et ál. (2006) y que comprende 5 fases de trabajo:

a) Precontemplación, que permitirá al individuo tomar conciencia de las necesidades.

b) Reflexión: a través de ella la persona puede autoanalizar sus posibles carencias, ordenar ideas y planificar.

c) Descubrimiento, para detectar creencias, el nivel de compromiso, etc.

d) Plan de acción, en el que hay que comprometerse con unos objetivos concretos, realistas, personales y motivadores.

e) Hábito, fase en la que se ha de producir transferencia de aprendizajes, de modo que se alcance la ejecución de forma autónoma.

A lo largo de estas fases se han de trabajar las facetas y dimensiones de la inteligencia espiritual (cognitivo, emocional y conductual) descritas por Arias y Lemos (2015), así como las fortalezas y virtudes humanas descritas por Peterson y Park (2009) y Castro (2010). 
A continuación, presentamos una descripción de la propuesta de coaching espiritual en la que estamos trabajando, basándonos en las fases anteriores y en los resultados del trabajo de Álvarez y Gudiño (2018).

\subsubsection{Fase 1: Exploración.}

En esta etapa, el coachee debe tomar conciencia de sus necesidades a nivel espiritual, está relacionada con la dimensión cognitiva, trabajando facetas como el sistema de valores, la búsqueda de su vocación o misión en la vida, etc. El coach debe provocar el razonamiento, por tanto, no se trata de buscar respuestas, sino de encontrar las preguntas que le van a permitir guiar "acompañar" al coachee en esa búsqueda de respuestas.

\subsubsection{Fase 2: Reflexión.}

Aquí el coach debe hacer de espejo frente a las respuestas del coachee, de manera que refleje sus palabras y pueda conocer mejor sus pensamientos, emociones, propósitos, es decir, conocerse mejor a sí mismo (autoconocimiento), de modo que pueda detectar sus carencias, ordenar ideas y planificar. En esta etapa se trabaja también la dimensión cognitiva de la inteligencia espiritual, pudiendo utilizar para ello la práctica de la meditación y la capacidad de mirar más allá (autotrascendencia).

\subsubsection{Fase 3: Descubrimiento.}

Esta fase iría unida a la anterior, pero está más relacionada con la dimensión afectiva, pues a medida que el coachee reflexiona sobre las posibles carencias 0 limitaciones, va detectando sus potencialidades y creencias, comprendiendo la relación de estas con su nivel de compromiso y puede ir estableciendo vínculos afectivos que le motiven a actuar. Para ello se deben analizar tanto los aspectos y experiencias positivas como los negativos, utilizando para ello el diálogo socrático.

\subsubsection{Fase 4: Planificación}

Esta fase iría unida a la anterior, pero está más relacionada con la dimensión afectiva, pues a medida que el coachee reflexiona sobre las posibles carencias 0 limitaciones, va detectando sus potencialidades y creencias, comprendiendo la relación de estas con su nivel de compromiso y puede ir estableciendo vínculos afectivos que le motiven a actuar.

\subsubsection{Fase 5: Actuación}

En esta fase se trabaja fundamentalmente la dimensión conductual, control de impulsos, sencillez, manejo de ocio, resistencia, etc. Para alcanzarlo es importante la transferencia de los aprendizajes realizados en las etapas anteriores y debe tener como finalidad el convertir este proceso en un hábito.

A lo largo de estas fases, se pretende que el coachee tome conciencia de su dimensión espiritual, genere un proyecto de vida coherente con sus valores, que le permita gozar de la misma y establecer lazos afectivos auténticos y profundos, 
influyendo también en su dimensión emocional, fomentado el crecimiento personal y el desarrollo integral de su persona. Para ello debe tener un compromiso con el proceso.

Este proceso de coaching solo se puede llevar a cabo por un coach que esté formado y preparado para ello, que tenga las competencias personales necesarias y que además conozca las diferentes herramientas tanto internas como externas que le permitan trabajar cada una de estas etapas. Esta es la fase en la que nos encontramos ahora, tras la fundamentación teórica, estamos trabajando en la generación de la formación necesaria para nuestro profesorado, respondiendo así al reto de formar docentes que sean competentes no solo en el ámbito cognitivo, sino también en el emocional y espiritual, permitiendo el desarrollo íntegro de la persona como ser bio-psico-socio-espiritual, como objetivo educativo.

\section{DISCUSIÓN Y CONCLUSIONES}

Comenzábamos este texto exponiendo la necesidad de trabajar las capacidades emocionales y espirituales para permitir el desarrollo integral de la persona, como reto educativo para mejorar la calidad de la enseñanza y el aprendizaje.

A raíz de la revisión bibliográfica realizada, hemos puesto de manifiesto no solo la existencia de la espiritualidad humana, sino la posibilidad de la existencia de una inteligencia espiritual susceptible de ser educada respondiendo así a las necesidades humanas de orden espiritual que nos hacen preguntarnos 0 plantearnos problemas existenciales como cuál es el significado de nuestra vida o el destino final del mundo.

Siguiendo la concepción humana planteada por la tercera fuerza psicológica, la psicología humanista, como un ser bio-psico-socio-espiritual, el ser humano tiene una existencia que trasciende a lo racional, y la incorporación de la inteligencia espiritual nos permite comprender su potencial y cómo alcanzar su pleno desarrollo. Es por esto que nos hemos acogido al modelo de inteligencia espiritual de Arias y Lemos (2015) que integra las dimensiones cognitiva, emocional y espiritual, haciéndolas dependientes unas de otras y ofreciéndonos una visión completa del ser humano. Además, lo hemos hecho converger con la propuesta de Peterson y Park (2009) y Castro (2010) que, basándose en la psicología positiva, consideran la espiritualidad como una de las fortalezas humanas que permiten el desarrollo pleno del potencial humano.

Para poder trabajar tanto la espiritualidad como la inteligencia espiritual, hemos optado por plantear un proceso de coaching, cuyo objetivo es el desarrollo de competencias, coincidiendo así con una de las finalidades del sistema educativo, el desarrollo máximo de las capacidades. Concretamente hemos propuesto un proyecto de coaching espiritual, en el que estamos trabajando, ya que está basado en la dialogo socrático, cuya base es la búsqueda de respuestas, coincidiendo así con la base del crecimiento espiritual. Pensamos que este trabajo de coaching espiritual permitirá incrementar la inteligencia espiritual y poder afrontar y resolver problemas de tipo existencial, como buscar el sentido de la vida, aumentar la 
capacidad de aceptar el sufrimiento, reflexionar, disfrutar del tiempo libre, sobreponerse a los sentimientos adversos o alcanzar un estado interno de estabilidad emocional.

\section{REFERENCIAS}

Álvarez, M. M. (2015). La Educación para la Interioridad como desafío Educativo. Tesis doctoral inédita. Universidad de Murcia.

Álvarez, L. A. y Gudiño, L. S. (2018). Coaching educativo: desarrollo de competencias en el educando de nivel superior. INNOVA Research Journal, 3(11), 169-182. https://doi.org/10.33890/innova.v3.n11.2018.804

Arias, R. y Lemos, V. (2015). Una aproximación teórica y empírica al constructo de inteligencia espiritual. Enfoques XXVII, 1, 79-102.

Barrón, R. (2010). Más allá del liderazgo empresarial: el coaching. Revista de la Facultad de Ciencias Contables, 17(34), 141-150.

Bayón, F., Cubeiro, J. C., Romo, M. y Sainz, J. A. (2006). Coaching realmente. Madrid: Pearson Prentice Hall.

Bisquerra, R. (2003). Educación emocional y competencias básicas para la vida. Revista de investigación Educativa, 3(1), 7-43.

Bisquerra, R. (2008). Coaching: un reto para los orientadores. Revista Española de Orientación y Psicopedagogía, 19(2), 163-170.

Bisquerra, R. (coord.) (2011). Educación emocional: propuesta para educadores y familias. Desclée.

Bustelo, J. L., Miró, A. P. y Barquero, J. D. (2018). La realidad de la Inteligencia Espiritual en las organizaciones españolas. Caso de estudio. European Journal of Applied Bussines Management Special Issue, 7(4), 41-56.

Carr, A. (2007). Psicología Positiva. La ciencia de la felicidad. Paidós.

Carril, J. (2008). Zen coaching: un nuevo método que funde la cultura oriental y occidental para potenciar al máximo tu vida profesional y personal. Díaz de Santos.

Castro, A. (comp.). (2010). Fundamentos de Psicología Positiva. Paidós.

Domínguez, R., Cruz, A. M. y Ferrando, A. M. (2018). Implementando el coaching educativo en la universidad virtual, una herramienta de desarrollo personal. Innoeduca. international journal of technology and educational innovation, 4(2), 150-158. http://dx.doi.org/10.24310/innoeduca.2018.v4i2.4930 
Gardner, H. (1995). Inteligencias múltiples. La teoría en la práctica. Paidós.

Gardner, H. (2011). La inteligencia reformulada. Las inteligencias múltiples en el siglo XXI. Paidós.

Goleman, D. (1995). Emotional Intelligence. Why it can matter more than IQ. Nueva York: Bantam Books. (Versión castellana: Inteligencia emocional. Kairós, 1996).

Palomera, R., Fernández-Berrocal, P. y Brackett, M. (2008). La inteligencia emocional como una competencia básica en la formación inicial de los docentes: algunas evidencias. Education y Psychology y editorial EOS.

Pérez, C. (2016). Inteligencia espiritual. Conceptualización y cartografía psicológica. INFAS Revista de psicología, 2(1), 63-70. ISSN: 0214-9877.

Peterson, C., y Park, N. (2009). El estudio científico de las fortalezas humanas. En C. Vázquez y G. Hervás, La ciencia del bienestar (pp. 181-207). Alianza.

Ramos, E.; Sierra, B. y Roces, C. (2019). Ámbitos de aplicación del Coaching educativo: una revisión bibliográfica del periodo 2013-17. Education Siglo XXI, 37(2), 223-244.

Salgado-Lévano, A. C. (2015). Formación universitaria en Psicología de la Religión y la Espiritualidad: ¿necesidad o utopía? Revista Digital de Investigación y Docencia Universitaria, 9(2), 89-113. ISNN: 223-2516

Sánchez, B. (2009). Bienestar espiritual en personas con y sin discapacidad. AQUICHAN, 9(1), 8-22.

Sánchez Mirón, B. y Boronat Mundina, J. (2014). Coaching Educativo: Modelo para el desarrollo de competencias intra e interpersonales. Educación $X X 1,17(1), 221$ 242. doi: http://dx.doi.org/10.5944/educxx1.17.1.10712

Torralba, F. (2018). Inteligencia Espiritual. 11ํe ed. Plataforma editorial.

Vargas, F. y Moya, L. (2018). La Espiritualidad como fortaleza humana y su relación con la construcción de sentido vital. Algunas notas específicas para el campo educativo. CAURIENSIA, Vol. XIII. 277-299. ISSN: 1886-4945.

Whitmore, J. (2016). Coaching. El método para mejorar el rendimiento de las personas. Paidós.

Woolfolk, A. (2010). Psicología Educativa. 11ํed. Pearson.

Zohar, D. y Marshall, I. (2001) Inteligencia Espiritual. Plaza \& Jané. 


\section{AUTORA}

\section{Elena Alarcón Orozco}

Doctora por la Universidad de Málaga y Licenciada en Psicología. Profesora Titular en el Departamento de Psicología del centro Adscrito de Magisterio Mํㅡㄴ Inmaculada de la Universidad de Málaga. Desarrolla su labor docente en la formación de Maestros de Infantil y Primaria. Su trayectoria investigadora se centra en la innovación educativa y la atención a la diversidad, concretamente en el desarrollo de metodologías activas, centradas en el alumno y en la resolución de conflictos en el aula, derivados del trabajo en equipo. Ha trabajado en el desarrollo de diversos Proyectos de Innovación educativa, entre ellos en el PIE13-067: Atención a la diversidad programando a partir de las Inteligencias múltiples. Es miembro del equipo de investigación "Grupo FORMACIÓN, Orientación, Empleabilidad, Emprendimiento, inclusión e Innovación" (FOREMPIN) (HUM-365) de la Universidad de Málaga.

elenaalarcon@eummia.es

Orcid ID: https://orcid.org/0000-0003-4021-1075 